\title{
Shockwave Coronary Lithoplasty for the Treatment of Under-Expanded Stent
}

Eliezer J. Tassone*, Cesare Tripolino, Gaetano Morabito, Placido Grillo, Bindo Missiroli

Department of Cardiac Surgery, Cardiology Unit, Sant'Anna Hospital-Catanzaro, Italy

Article Info

\section{Article Notes}

Received: June 26, 2020

Accepted: July 17, 2020

\section{${ }^{*}$ Correspondence:}

Dr. Eliezer Joseph Tassone, Via Paolo Orsi, 5/7 88100 Catanzaro, Italy; Telephone No: 39 3298861912; Email: eliezerjoseph.tassone@gmail.com.

(c) 2020 Tassone EJ. This article is distributed under the terms of the Creative Commons Attribution 4.0 International License.

\section{Keywords:}

Coronary calcification

Atherectomy

Under-expanded stent

Coronary angioplasty

Shockwave

Coronary lithotripsy

\section{Abstract}

The presence of coronary calcification is a hard challenge for the interventional cardiologist, as it is associated with incomplete stent expansion and frequently stent failure. In recent years, innovative techniques have been developed to treat coronary calcific lesions such as rotational atherectomy. However, many of them are burdened with an increased procedural risk. Recently, a new technique called "Shockwave Coronary Lithoplasty System", also called lithotripsy, has been introduced in order to treat calcific coronary lesions with greater safety. Shockwave procedure allows treating the most calcific coronary lesions with simplicity and safety. This system employs the sound waves, similar to that used for treating kidney stones, in order to crush the calcific lesions. In this minireview, we explain the characteristics of the method and we provide a description of the technique in detail on the basis of the preliminary experience of the first cases. In particular, we will demonstrate that this technique is more effective and safer than traditional techniques employing atherectomy, also providing for the first time a therapeutic chance for the treatment of under-expanded stents in many clinical contexts.

\section{Introduction}

Coronary artery calcifications (CAC) represent a challenge to the interventional cardiologist, as they are often hard to treat with conventional balloon angioplasty ${ }^{1}$. The prevalence of CAC is increasing as well as their impact on PCI procedures and outcomes. In fact, several risk factors as well as chronic inflammatory conditions, lead to calcium deposition in the coronary arteries. All these conditions cause endothelial damage and subsequent inflammatory response, which are the promoters of calcium deposition in the arterial wall. On the other hand, because intravascular imaging techniques, such as intravascular ultrasound (IVUS) and optical coherence tomography are used not routinely, CAC is often underestimated ${ }^{2}$. It is known that the presence of CAC is associated with incomplete and/or asymmetrical stent expansion as well as lower rates of procedural success, greater risk of angiographic complications, and a greater prevalence of subsequent adverse events ${ }^{3}$. Moreover, CAC represents a risk factor for stent deformation because they associate with a reduction in drug concentration and uptake into the vessel wall from the stent in addition to its underexpansion ${ }^{4,5}$.

Current techniques to debulk calcific stenosis include standard or high-pressure non-compliant balloons, cutting/scoring balloons, or atherectomy. Recent evidence indicates that coronary atherectomy is utilized in less than $5 \%$ of PCI patients compared to a CAC prevalence of $30 \%$. Therefore, most patients with CAC is still treated with Plain Old Balloon Angioplasty (POBA) and Drug Eluting Stent (DES). In fact, this technique may lead to vascular wall 
injury and coronary dissection or perforation ${ }^{5,6}$. Recently, therapeutic armamentarium has been enriched by the coronary lithotripsy ${ }^{6,7}$. It consists of a novel technology combining a balloon angioplasty catheter with the use of sound waves, similar to that used for treating kidney stones. Lithotripsy catheter emits sound waves aimed to disrupt arterial calcifications before stent implantation. Preliminary evidence suggest that it is a sure and reliable tool to overcome calcified stenosis in the coronary tree.

\section{Description}

We previously described the first cases of patients with stent under-expansion due to a pre-existing calcium depots successfully treated with the shockwave coronary lithotripsy system ${ }^{8,9}$. Briefly, we reported the case of a 60-year-old Caucasian man with the clinical history of primary coronary angioplasty (PCI) and stenting of ramus intermedius coronary artery (RI) performed about one month ago in another Center. An angiographic control study was performed after 1 month that revealed a critical calcific re-stenosis of RI with the evidence at IVUS study of the under-expansion of previously implanted DES due to calcium depots. After group discussion, in consideration of the difficulty of performing a stenting procedure in the previous calcific stent as well as the risk of rotational atherectomy, lithotripsy-enhanced disruption of calcium beyond the stents was performed with the coronary lithotripsy. We previously reported another case of a 77-year-old Caucasian woman successfully treated with coronary lithotripsy in which angiographic study revealed stent under-expansion at its proximal edge and critical stenosis in medium and distal traits of left anterior descending artery (LAD). Also, in this case IVUS study helped us to better characterize the lesion at the stent level, confirming the stent under-expansion due to heavily calcified plaque ${ }^{9}$. After these descriptions, other cases were reported that showed the use of lithotripsy fort the treatment of under-expanded stent. Tovar Forero et al. reported the case of a 74-year old man undergoing coronary angiography for class III angina ${ }^{10}$. Angiographic study showed the presence of a great-calcified stenotic lesion that compromised the DES implantation despite the utilization of post-dilatation non-compliant balloon at high-pressure. Thus, the decision to use a shockwave balloon was successfully made, confirming the useful and safety of lithotripsy also for the under-expanded stent. Alfonso et al. recently described the case of a 63-year old man with acute coronary syndrome due to severe, heavily calcified, new lesion in a large obtuse marginal branch and in-stent restenosis in the left anterior descending coronary artery previously treated about 15 years ago ${ }^{11}$. Several conventional and noncompliant balloons at very high pressure such as the use of different scoring balloons failed to dilate the lesions. Therefore, coronary lithotripsy was successfully used to dilate both the lesions before DES implantation as demonstrated by the excellent angiographic and OCT result. Recently we reported another case that confirms the usefulness and safety of coronary lithotripsy also in the context of ST elevation myocardial infarction ${ }^{12}$. An 80-year-old Caucasian man with ST elevation myocardial infarction underwent emergent coronary angiography showing complete intrastent thrombosis at the proximal trait of LAD. After thrombus removal, it was evident that stent under-expansion at its proximal edge was caused by vascular calcification. Coronary lithotripsy allowed to treat this lesion. After calcium deposits disruption, we were able to obtain complete stent expansion.

Our initial experience encourages us to use coronary lithotripsy in all conditions in which the presence of coronary calcification does not allow correct stent implantation. Furthermore, the lithotripsy has proved to be safe and effective for the treatment of under-expanded stents. Below we present our experience in the use of Shockwave coronary lithotripsy to treat the underexpanded stents providing furthermore an explanation of the employed method. In table 1 are reported the clinical

Table 1. Clinical characteristics of patients.

\begin{tabular}{|l|c|c|c|c|c|c|c|c|c|c|c|}
\hline \multicolumn{1}{|c|}{ Patients } & $\mathbf{1}$ & $\mathbf{2}$ & $\mathbf{3}$ & $\mathbf{4}$ & $\mathbf{5}$ & $\mathbf{6}$ & $\mathbf{7}$ & $\mathbf{8}$ & $\mathbf{9}$ & $\mathbf{1 0}$ & Means \pm SD \\
\hline Age (years) & 60 & 77 & 65 & 66 & 80 & 62 & 59 & 57 & 69 & 63 & $65.8 \pm 7.2$ \\
\hline Gender & Male & Female & Male & Male & Male & Male & Male & Male & Male & Male & - \\
\hline Hypertension & Yes & Yes & Yes & Yes & Yes & Yes & Yes & Yes & Yes & Yes & - \\
\hline Diabetes & None & Yes & Yes & Yes & Yes & Yes & None & Yes & Yes & None & - \\
\hline Dyslipidaemia & Yes & Yes & Yes & Yes & Yes & Yes & Yes & Yes & Yes & Yes & - \\
\hline Smoker & Yes & None & None & Yes & Yes & None & None & None & Yes & None & - \\
\hline History of MI & Yes & Yes & Yes & None & Yes & None & Yes & Yes & Yes & Yes & - \\
\hline History of CABG & None & None & None & None & None & None & None & None & None & None & - \\
\hline History of AF & None & Yes & None & None & None & Yes & None & None & None & None & - \\
\hline History of PAD & None & None & Yes & None & None & Yes & None & None & None & None & - \\
\hline LVEF & $60 \%$ & $45 \%$ & $55 \%$ & $50 \%$ & $45 \%$ & $50 \%$ & $45 \%$ & $55 \%$ & $50 \%$ & $60 \%$ & $51.5 \pm 5.5$ \\
\hline SA at admission & Yes & Yes & Yes & Yes & Yes & Yes & None & Yes & Yes & Yes & - \\
\hline
\end{tabular}

MI: myocardial infarction; CABG: Coronary Artery Bypass Graft; AF: Atrial Fibrillation; PAD: Peripheral Artery Disease; LVEF: Left Ventricular Ejection Fraction; SA: Stable Angina. 
characteristics of 10 patients examined. In particular, 8/10 patients were diabetics, all patients had dyslipidemia and arterial hypertension; $8 / 10$ patients had history of myocardial infarction, all patients had stable angina at recovery; echocardiographic examination revealed a preserved function for most of them. Table 2 shows the characteristics of the coronary lesions found during the first angiographic study performed a few years ago and the subsequent procedures (included type, length and diameter of stents utilized, as well as the balloon characteristics). Finally, in table 3 the procedures performed during lithotripsy are reported. In particular, angiographic study revealed a critical re-stenosis in the site of previous stent implantation. IVUS study confirmed the presence of under-expansion of previously implanted DES due to calcium depots with a significant reduction of the Minimal Lumen Area (MLA). In detail, a range of 3.0-4.0 x $12 \mathrm{~mm}$ lithotripsy balloon (Shockwave Medical, Fremont, California) was used to pre-dilate and treat the entire length of disease. It was inflated to 4-12 atm range, with 2-8 cycles of ultrasound energy of 10 seconds. Once the lithotripsy treatment was completed, a non-compliant

Table 2. Lesions and procedural characteristics at first angiographic study.

\begin{tabular}{|l|c|c|c|c|c|c|c|c|c|c|c|}
\hline \multicolumn{1}{|c|}{ Patients } & $\mathbf{1}$ & $\mathbf{2}$ & $\mathbf{3}$ & $\mathbf{4}$ & $\mathbf{5}$ & $\mathbf{6}$ & $\mathbf{7}$ & $\mathbf{8}$ & $\mathbf{9}$ & $\mathbf{1 0}$ & $\mathbf{M e a n s} \pm$ SD \\
\hline Date of first PCI & $15 / 05 / 18$ & $19 / 12 / 13$ & $12 / 8 / 14$ & $18 / 6 / 15$ & $13 / 02 / 17$ & $22 / 05 / 18$ & $13 / 03 / 18$ & $21 / 07 / 15$ & $05 / 07 / 2018$ & $15 / 04 / 2018$ & - \\
\hline Target lesion & RI & LAD & LAD & LAD & LAD & LAD & CX & LAD & RCA & LAD & - \\
\hline Stent type & Onyx & Resolute & Synergy & Onyx & Onyx & DES & Onyx & DES & DES & Resolute \\
\hline Stent diameter (mm) & 3 & 2.5 & 2.75 & 3.5 & 3 & 3 & 2.75 & 3 & 3 & 3 & $2.95 \pm 0.24$ \\
\hline Stent length (mm) & 18 & 26 & 24 & 15 & 22 & 22 & 20 & 20 & 20 & 20 & $20.7 \pm 2.9$ \\
\hline Atm & 18 & 16 & 16 & 20 & 18 & 22 & 18 & 20 & 20 & 20 & $18.8 \pm 1.8$ \\
\hline Postdilatation NCb & Yes & Yes & Yes & Yes & Yes & Yes & Yes & Yes & Yes & Yes & - \\
\hline $\begin{array}{l}\text { NC balloon diameter } \\
\text { (mm) }\end{array}$ & 3.5 & 2.75 & 3.25 & 3.75 & 3.5 & 3.25 & 3 & 3.25 & 3.5 & 3.5 & $3.32 \pm 0.27$ \\
\hline $\begin{array}{l}\text { NC balloon length } \\
\text { (mm) }\end{array}$ & 15 & 15 & 15 & 15 & 15 & 15 & 15 & 15 & 15 & 15 & 15 \\
\hline Atm & 20 & 16 & 20 & 24 & 20 & 20 & 22 & 20 & 20 & 20 & $20.2 \pm 1.9$ \\
\hline
\end{tabular}

$\mathrm{NC}=$ non-compliant; Atm= atmosphere; RI: Ramus Intermedius; LAD: Left Anterior Descending Artery; CX=Circumflex coronary artery; RCA: Right Coronary Artery; DES: Drug Eluting Stent (unspecified)

Table 3. Lesions and procedural characteristics of ShockWave Lithoplasty.

\begin{tabular}{|c|c|c|c|c|c|c|c|c|c|c|c|}
\hline Patients & 1 & 2 & 3 & 4 & 5 & 6 & 7 & 8 & 9 & 10 & Means \pm SD \\
\hline Date of Lithotripsy & $19 / 06 / 18$ & $11 / 07 / 18$ & $30 / 11 / 18$ & $10 / 12 / 18$ & $22 / 03 / 2019$ & $24 / 05 / 2019$ & $31 / 05 / 2019$ & $03 / 09 / 2019$ & $05 / 11 / 2019$ & 05/11/2019 & - \\
\hline Angio-stenosis (\%) & 85 & 80 & 80 & 75 & 100 & 70 & 70 & 70 & 80 & 75 & $78.5 \pm 8.6$ \\
\hline Pre-IVUS MLA (mmq) & 3.2 & 2.86 & 4.2 & l & 2.05 & / & 3.45 & 4.76 & 4.39 & 3.89 & \\
\hline Predilatation & None & None & None & Yes & None & None & None & Yes & Yes & None & - \\
\hline $\begin{array}{l}\text { Lithotripsy balloon } \\
\text { diameter }(\mathrm{mm})\end{array}$ & 3 & 3 & 3.5 & 3.5 & 4 & 3.5 & 3.5 & 3.5 & 3.5 & 3 & $3.60 \pm 0.83$ \\
\hline $\begin{array}{l}\text { Lithotripsy balloon } \\
\text { lenght }(\mathrm{mm})\end{array}$ & 12 & 12 & 12 & 12 & 12 & 12 & 12 & 12 & 12 & 12 & 12 \\
\hline Lithotripsy pulses & 30 & 20 & 40 & 80 & 70 & 80 & 80 & 80 & 70 & 80 & $63 \pm 22$ \\
\hline $\begin{array}{l}\text { Lithotripsy balloon } \\
\text { inflation (atm) }\end{array}$ & 6 & 6 & 4 & 6 & 10 & 6 & 10 & 10 & 10 & 12 & $8 \pm 2.5$ \\
\hline Postdilatation & Yes & Yes & Yes & Yes & Yes & Yes & Yes & Yes & Yes & Yes & - \\
\hline $\begin{array}{l}\text { NC balloon diameter } \\
(\mathrm{mm})\end{array}$ & 3.25 & 3 & 3.5 & 3.5 & 4 & 3 & 3.5 & 3.5 & 3.5 & 3.5 & $3.42 \pm 0.27$ \\
\hline $\mathrm{NC}$ balloon lenght $(\mathrm{mm})$ & 15 & 15 & 21 & 15 & 21 & 15 & 15 & 15 & 15 & 21 & $16.8 \pm 2.7$ \\
\hline Atm & 12 & 18 & 20 & 14 & 14 & 16 & 12 & 20 & 20 & 20 & $16.6 \pm 3.2$ \\
\hline $\begin{array}{l}\text { DES/DEB implantation } \\
\text { after Lithotripsy }\end{array}$ & None & None & DES & DES & DES & None & DEB & None & None & None & - \\
\hline DES type & l & l & Synergy & Synergy & Synergy & I & DEB Agent & l & l & l & - \\
\hline DES diameter (mm) & I & / & 3.5 & 4.0 & 3.5 & I & 3.0 & I & l & l & $3.5 \pm 0.35$ \\
\hline DES lenght $(\mathrm{mm})$ & l & l & 48 & 20 & 48 & l & 30 & l & l & l & $36.5 \pm 12.0$ \\
\hline Atm & / & / & 22 & 22 & 22 & / & 22 & / & l & l & 22 \\
\hline Post- IVUS MLA (mm) & 7.47 & 6.37 & 7.83 & 14.93 & 7.45 & 8.25 & 7.87 & 9.30 & 7.67 & 6.95 & $8.40 \pm 2.29$ \\
\hline IVUS- MLA gain (mm) & 4.27 & 3.51 & 3.63 & I & 5.40 & I & 4.42 & 4.54 & 3.28 & 3.06 & $4.01 \pm 0.73$ \\
\hline IVUS- MLA gain (\%) & 133 & 122 & 86 & l & 263 & l & 128 & 95 & 75 & 79 & $122.6 \pm 57.1$ \\
\hline
\end{tabular}

Atm: Atmosphere; IVUS=intravascular ultrasound; MLA= minimal lumen area; NC= non-compliant; DES: Drug Eluting Stent; DEB: Drug-Eluting Ballon; IVUS: IntraVascular UltraSound; MLA: Minimum Lumen Area. 
balloon (Apollo $^{\mathrm{TM}}$ range 3.0-4.0 x 15-21 mm, Skyler Scientific, Malaysia) inflated at 12-20 atm was used to postdilate the stent struts. IVUS after cycles of "Shockwave" showed significant area gain $>75 \%$. In $3 / 10$ patients the procedure was completed with DES implantation after lithotripsy, 1 patient underwent drug-eluting balloon treatment. Excellent post procedure angiographic result was obtained (TIMI 3), as well as the MLA on IVUS final control (figure 1 and 2). Patients were discharged in good condition and without symptoms. All patients one month after discharge underwent to a cardiologic control that included clinical examination, electrocardiogram and echocardiography. Nobody presented episodes of MACE, including myocardial infarction, stroke, and death; we also excluded new hospitalization for angina, acute coronary syndrome and heart failure.

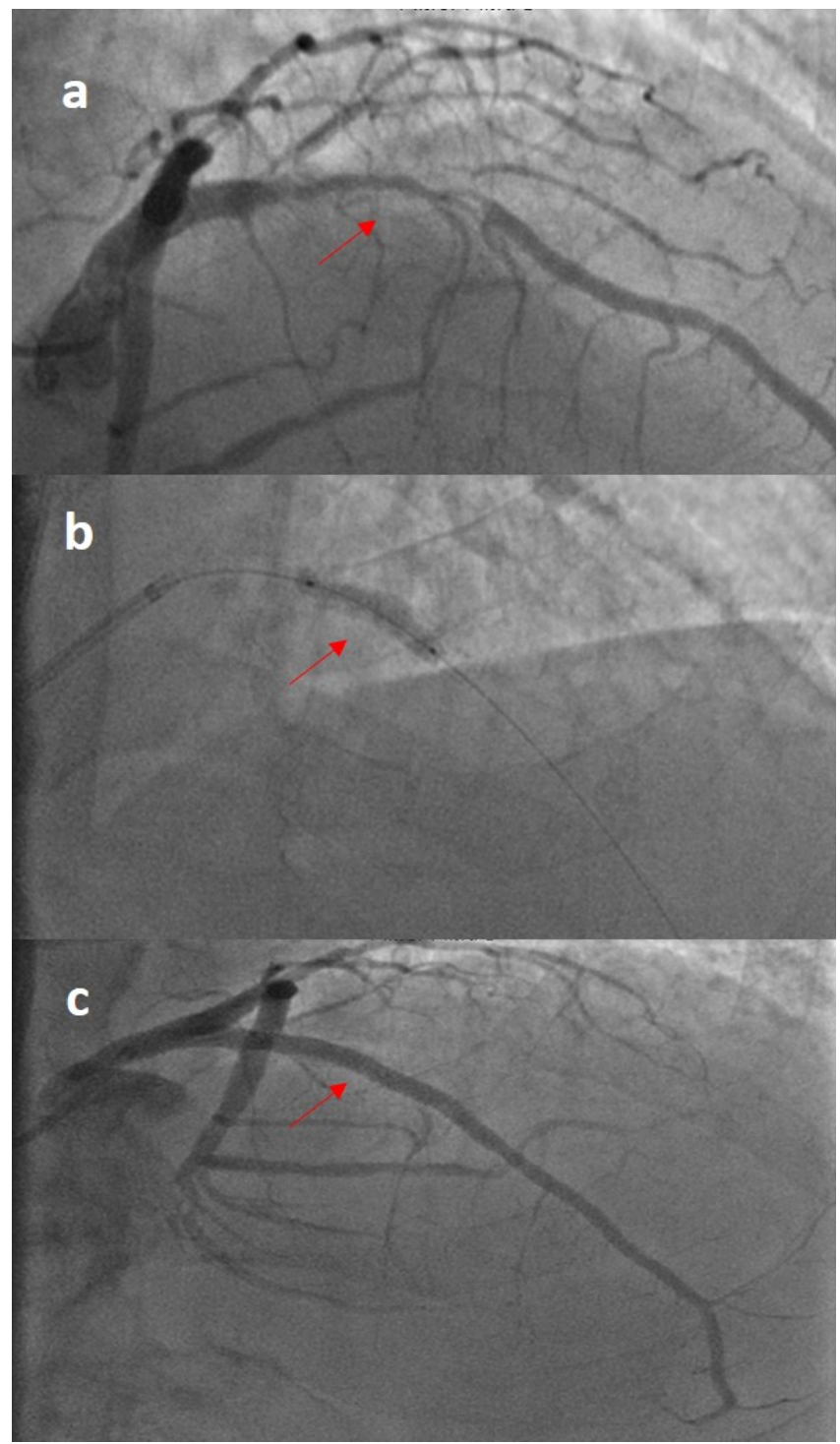

Figure 1. Long critical stenosis of LAD (red arrow) due to calcific plaque (A) treated with lithotripsy (B) and stent implantation; the angiographic result showed in the panel $\mathrm{C}$.

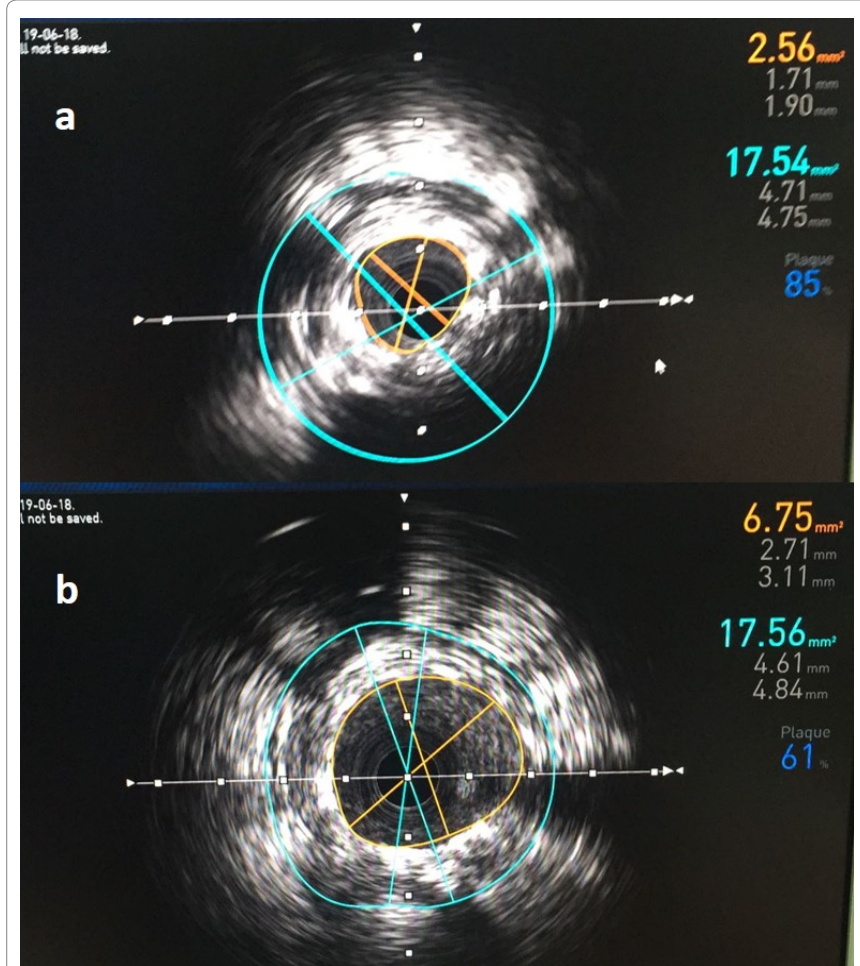

Figure 2. IVUS study before (A) and after (B) cycles of "Shockwave" during lithotripsy showed significant increase in Minimal Lumen Area (on the top right, $2.56 \mathrm{~mm}^{2}$ vs $6.75 \mathrm{~mm}^{2}$ ).

\section{Discussion}

We reported some of the few cases in which the "Shockwave" method is used ${ }^{13}$. In particular, we describe the first cases in which this method is used to treat a calcified re-stenosis in a site of previous stenting. Present data confirm that Shockwave procedure allows the most calcific coronary lesions to be treated with simplicity and safety also in case of stent under-expansion. These lesions are very hard to treat because calcium deposits wrapping the stents does not allow a correct expansion. The lithotripsy with Shockwave System is a novel technology that uses the principle of lithotripsy, already widely used in urology for the treatment of kidney stones. The system consists of a balloon catheter, equipped with lithotripsy emitters positioned on the useful length of the balloon, and a generator (connected to the catheter with a connecting cable) that is used to activate, by means of a button, the lithotripsy cycles and therefore the emission of shock waves that, in practice, weaken calcium linkage and enhances plaque compliance. Once the calcium has been modified, the vessel can be dilated using low pressure. The lithotripsy system received FDA approval for the treatment of peripheral arteries in 2016. Its main use is finalized to treat calcified lesions in several vascular districts, such as iliac, femoral, popliteal, and renal arteries ${ }^{14,15}$. The novelty of these data consists in the use of Shockwave to treat the calcified re-stenosis of the previously treated lesions in a 
site of under-expanded stent. In these cases, the lithotripsy system to be the only possible method to safely treat the lesions because they represent the calcific re-stenosis in the context of previous stents, which therefore contraindicate other preparatory procedures such as rotational atherectomy (Rotablator), a system that pulverizes the calcific plaque, increasing however the procedural risk. The less invasiveness and the greater safety of this method, compared to others that use traditional atherectomy, could encourage its use in the limit situations in which the contraindication to surgery represents an obstacle to the treatment of coronary stenosis, with the risk that many of these patients remain untreated and without any potential benefit of revascularization therapy. A wider diffusion of the lithotripsy would allow studying its effects in the short and long term on samples of a larger population in order to be able to validate its use in the common interventional practice.

\section{Conclusion}

Our experience demonstrates for the first time that lithotripsy might represent a new useful tool for the treatment of stent under-expansion secondary to calcified plaque.

\section{References}

1. Kobayashi Y, Okura H, Kume T, et al. Impact of target lesion coronary calcification on stent expansion. Circ J. 2014; 78: 2209-2214.

2. Fitzgerald PJ, Oshima A, Hayase M, et al. Final results of the Can Routine Ultrasound Influence Stent Expansion (CRUISE) study. Circulation. 2000; 102: 523-530.

3. Mintz GS. Intravascular imaging of coronary calcification and its clinical implications. JACC Cardiovasc Imaging. 2015; 8: 461-471.

4. Ahn JM, Kang SJ, Yoon SH, et al. Intravascular ultrasound findings of early stent thrombosis after primary percutaneous intervention in acute myocardial infarction: a Harmonizing Outcomes with Revascularization and Stents in Acute Myocardial Infarction (HORIZONS-AMI) substudy. Circ Cardiovasc Interv. 2011; 4: 239-247.

5. Ahn JM, Kang SJ, Yoon SH, et al. Meta-analysis of outcomes after intravascular ultrasound-guided versus angiography-guided drugeluting stent implantation in 26,503 patients enrolled in three randomized trials and 14 observational studies. Am J Cardiol. 2014; 113: $1338-1347$.

6. Ali ZA, Brinton TJ, Hill JM, et al. Optical Coherence Tomography Characterization of Coronary Lithoplasty for Treatment of Calcified Lesions: First Description. JACC Cardiovasc Imaging. 2017; 10: 897-906.

7. De Silva K, Roy J, Webb I, et al. A calcific, undilatable Stenosis: Lithoplasty, a New Tool in the Box? JACC Cardiovasc Interv. 2017; 10: 304-306.

8. Tassone EJ, Tripolino C, Morabito G, et al. When Calcium Gets Tough, the Tough Cardiologist Starts to Play... Cardiology. 2019; 14: 167-171.

9. Morabito G, Tripolino C, Tassone EJ, et al. A Case of Stent UnderExpansion due to Calcified Plaque Treated with Shockwave Lithoplasty. Cardiology. 2018; 141: 75-77.

10. Tovar Forero MN, Wilschut J, Van Mieghem NM, et al. Coronary lithoplasty: a novel treatment for stent underexpansion. Eur Heart J. 2019; 40: 221 .

11. Alfonso F, Bastante T, Antuna P, et al. Coronary lithoplasty for the treatment of undilatable calcified de novo and in-stent restenosis lesions. JACC Cardiovasc Interv. 2019; 12: 497-499.

12. Tripolino C, Tassone EJ, Morabito G, et al. ST-elevation myocardial infarction due to stent underexpansion managed with coronary lithoplasty. Rev Recent Clin Trials. 2019; 14: 292-295.

13. Ali ZA, Nef H, Escaned J, et al. Safety and Effectiveness of Coronary Intravascular Lithotripsy for Treatment of Severely Calcified Coronary Stenoses: The Disrupt CAD II Study. Circ Cardiovasc Interv. 2019; 12: e008434.

14. Brodmann M, Holden A, Zeller T. Safety and Feasibility of Intravascular Lithotripsy for Treatment of Below-the-Knee Arterial Stenoses. J Endovasc Ther. 2018; 25: 499-503.

15. Brodmann M, Werner M, Brinton TJ, et al. Safety and Performance of Lithoplasty for Treatment of Calcified Peripheral Artery Lesions. J Am Coll Cardiol. 2017; 70: 908-910. 